\title{
Uso del "ChemSensor" como herramienta de discriminación precoz del semen de cerdo lbérico en función de su congelabilidad
}

\author{
J. Gómez-Fernández ${ }^{1}$, C. Tomás², J.A. Carrasco³, R. Sánchez-Sánchez ${ }^{4}$, \\ A. González-Bulnes ${ }^{4}$, E. Gómez-Izquierdo ${ }^{1}$ y E. de Mercado ${ }^{1, *}$ \\ 1 Centro de Pruebas de Porcino. Área de Investigación Ganadera. Subdirección de Investigación y Tec- \\ nología. Instituto Tecnológico Agrario. Consejería de Agricultura y Ganadería. Junta de Castilla y \\ León. España. Ctra Riaza-Toro s/n, 40353 Hontalbilla, Segovia, España \\ 2 Centro de Investigación y Tecnología Animal (CITA), Instituto Valenciano de Investigaciones Agrarias \\ (IVIA). Apdo. 187. 12400- Segorbe (Castellón), España \\ 3 Instituto de Ciencia y Tecnología de Alimentos y Nutrición (ICTAN), C/José Antonio Novais, 10, 28040 \\ Madrid, España. \\ 4 Dpto. de Reproducción Animal, INIA, Avda. Puerta de Hierro s/n, 28040 Madrid, España
}

\section{Resumen}

Para favorecer el uso de dosis de inseminación de semen de porcino congelado-descongelado a nivel comercial, sería interesante conocer de antemano si el semen de verraco congelará bien o mal. El objetivo de este estudio fue evaluar el "ChemSensor" (cromatógrafo de gases-masas unido a un software de análisis quimiométrico) como herramienta precoz de discriminación entre eyaculados según su congelabilidad. Para ello se utilizaron 33 eyaculados de verracos de raza lbérica de los cuales se cogió una alícuota de $1 \mathrm{ml}$ de semen y se analizó con el "ChemSensor", el resto del eyaculado fue congelado-descongelado usando un protocolo estandarizado, para determinar su congelabilidad (buenos y malos congeladores) en función del porcentaje de espermatozoides con la membrana plasmática intacta y el porcentaje de espermatozoides móviles totales post-descongelación. Para el análisis con el "ChemSensor", las muestras se volatilizaron e ionizaron descomponiéndolas en diferentes iones con un tamaño determinado, que se usaron como variables de discriminación, para separar los eyaculados en función de su congelabilidad. El "ChemSensor" fue capaz de discriminar todos los eyaculados de los buenos congeladores; y dentro del grupo de los malos congeladores solamente una muestra fue clasificada erróneamente. Debido al reducido número de muestras, la distancia obtenida entre grupos $(2,62)$, aunque no muy elevada, podría considerarse como significativa, aunque sería necesario un mayor número de muestras para poder crear un modelo matemático más robusto. En conclusión, el "ChemSensor" es una posible herramienta adecuada para la discriminación precoz de eyaculados de cerdo lbérico en función de su congelabilidad.

Palabras clave: Congelación, Cromatografía gases-masas, compuestos volátiles del semen, semen cerdo ibérico.

* Autor para correspondencia: ita-merpened@itacyl.es http://dx.doi.org/10.12706/itea.2015.003 


\begin{abstract}
Use of "ChemSensor" as an early discrimination tool of Iberian pig semen according to their freezability

For the use of insemination dose of frozen-thawed boar sperm at the commercial level, it would be very interesting to know, in advance, if sperm from a boar will freeze well or poorly. So the aim of this study was to evaluate the ability of the "ChemSensor" (gas chromatograph-mass coupled with chemometric analysis software) as an early discrimination tool of Iberian pig ejaculates according to their freezability. For this, 33 ejaculates from Iberian breed boars were used and an aliquot of $1 \mathrm{ml}$ of semen from each one was analyzed with the "ChemSensor", the rest of the ejaculate was frozen-thawed using a standardized protocol to determine their freezability (good and bad freezers), depending on the percentage of intact plasma membrane and total motile post-thaw sperm. For analysis of "ChemSensor", sperm samples were volatilized and ionized, decomposing into different ions with a determined size, which were used as discrimination variables to determine if it is possible the separation of ejaculates into two freezability groups. The "ChemSensor" was able to discriminate all the ejaculates of the good freezers; and inside of bad freezers group only one sample was misclassified. Due to the small number of samples, the distance obtained between groups (2.62), although not very high, it could be considered significant, although a larger sample in order to create a more robust mathematical model would be necessary. In conclusion, the "ChemSensor" is a possible suitable tool for the early discrimination of Iberian pig ejaculates according to their freezability.
\end{abstract}

Key words: Freezing, gas chromatography-mass spectroscopy; volatile components, Iberian boar semen.

\section{Introducción}

La existencia de una elevada población de verracos cuyos espermatozoides muestran mala tolerancia permanente a la crioconservación, es un serio problema para la producción rentable de dosis espermáticas con este sistema de conservación, siendo uno los principales factores que limitan el diseño efectivo de protocolos de congelación (Medrano et al., 2002). Hasta ahora la selección de verracos ha estado basada en los rendimientos productivos que estos brindan a su descendencia, y no en la selección de reproductores por su capacidad de criopreservación espermática, como se viene haciendo durante años en el vacuno de leche (Vishwanath, 2003).

En la actualidad, los verracos suelen clasificarse principalmente en "buenos" y "malos" según su congelabilidad espermática, basándose principalmente en la motilidad y viabilidad de sus espermatozoides post-descongelación (Watson, 1995; Thurston et al., 1999; Gil et al., 2005; Hernández et al., 2007; Barranco et al., 2013). Esta clasificación se aplica a los verracos, y se establece en todos sus eyaculados, ya que se ha demostrado que la congelabilidad o susceptibilidad de que un eyaculado congele bien viene determinado genéticamente por el animal (Thurston et al., 2001), y por tanto es algo intrínseco del animal. Además estudios posteriores determinaron, si dentro de un mismo animal distintos eyaculados podrían tener distinta congelabilidad (Roca et al., 2006), determinándose de nuevo que es el verraco y no el eyaculado el que determina mayoritariamente si uno de sus eyaculados congelará bien o mal. Sin embargo, lo que se desconoce es cómo esas diferencias genéticas se expresan para que un eyaculado tenga una mayor o menor congelabilidad. Estas diferencias podrían estar representadas tanto por una distinta composición lipídica o proteica de la membrana espermática, como por variaciones en la composición del plasma seminal según la funcionalidad de las glándulas accesorias (Holt et al., 2005). 
El poder conocer de antemano si el semen de un verraco congelará bien o mal, sería un punto vital para el uso comercial de la técnica de crioconservación espermática, ya que se han correlacionado mayores niveles de fertilidad cuando se usa semen de buenos congeladores que cuando se usa de malos congeladores (Casas et al., 2010a). Además, el semen de los buenos congeladores tiene una mayor calidad, lo que permitiría mejorar notablemente las muestras congeladas en los bancos de conservación de especies en peligro de extinción, como sucede con algunas estirpes del cerdo Ibérico. Por ello, es necesario encontrar métodos que permitan la determinación precoz de la congelabilidad de los eyaculados, siendo el "ChemSensor" una posible herramienta eficaz en la búsqueda de este objetivo.

El "ChemSensor" o también Ilamado Sensor químico, es un cromatógrafo de gases-masas (GC-MS) unido a un software de análisis quimiométrico, que tiene numerosas aplicaciones en las industrias alimentaria, farmacéutica y química, en tareas de discriminación de diferente tipo de muestras (Radovic et al., 2001; Ansorena et al., 2001; Landaud et al., 2008; Rodríguez-Bencomo et al., 2009; Peris y Escuder-Gilabert, 2009). En este tipo de equipos las muestras a clasificar son volatilizadas, y aunque se obtiene un cromatograma con los diferentes compuestos volátiles, el análisis de clasificación lo realiza en base al espectro de masas específico de cada molécula, obtenido a partir de la fragmentación carga-masa iónica de todos los compuestos volátiles en conjunto (Busto et al., 2002; Armanino et al., 2008; Cynkar et al., 2010; Vera et al., 2010). El detector de masas del cromatógrafo fragmenta las moléculas mediante un impacto electrónico que produce una serie de fracciones másicas con unos valores $\mathrm{m} / \mathrm{z}$ (masa/carga) y con una abundancia determinada de cada una de las fracciones. La suma de las abundancias de cada masa iónica, de cada uno de los espec- tros, nos da una matriz de datos que puede superar los 500, pudiendo usar cada fracción másica como una verdadera variable para la clasificación. Para poder manipular este gran conjunto de datos es necesario recurrir al análisis multivariante mediante herramientas quimiométricas, como el método de reconocimiento de patrones SIMCA (Soft Independent Modelling Class Analogy) (Peris y Escuder-Gilabert, 2009). Este método permite asignar una clasificación previa al análisis de nuestras muestras (en este caso grupos de congelabilidad), separando cada uno de los grupos en función de la analogía que tienen cada una de las muestras que lo integran mediante un análisis de componentes principales (en este caso estos componentes serían las fracciones másicas), determinándose así un modelo para cada grupo de clasificación y estableciendo un límite entre las muestras pertenecientes a cada uno los grupos de estudio y las muestras que no pertenecen a ninguno, para un nivel de confianza de $95 \%$.

El uso combinado del "ChemSensor" con la técnica de análisis SIMCA ha sido recientemente puesto a punto por el Instituto de Ciencia y Tecnología de Alimentos y Nutrición (ICTAN) del CSIC, como una herramienta discriminatoria entre cerdos alimentados con diferentes dietas (Martín et al., 2009; Carrasco y Duque, 2013), a partir de la información aportada por el cromatograma de muestras de ácidos grasos, y compuestos volátiles de muestras de tocino.

Así pues, si esta técnica híbrida da la posibilidad de discriminar una muestra de un animal en función de una clasificación previa, cabe la posibilidad de poder discriminar entre muestras de semen de diferentes verracos, diferenciando entre "buenos" y "malos" congeladores, previa relación de los datos obtenidos por el sensor químico con su potencial de congelabilidad (evaluación de parámetros de calidad espermática tras la descongelación de las muestras). 
Por todo esto, el objetivo de este estudio fue determinar la capacidad del "ChemSensor" como herramienta de discriminación precoz del semen de cerdo lbérico en función de su congelabilidad.

\section{Material y métodos}

Recolección de los eyaculados

Se recogió un eyaculado de 33 verracos lbéricos sexualmente maduros procedentes de diferentes centros de inseminación. Los verracos tenían edades comprendidas entre los 2 y 3 años y estaban alojados en celdas individuales en un ambiente controlado (15-25 ${ }^{\circ} \mathrm{C}$ ) con exposición diaria a luz natural suplementada con luz artificial hasta las 16 horas de luz diarias. Los animales tuvieron acceso ad libitum al agua y fueron alimentados con una dieta comercial adecuada para los requerimientos nutricionales de un verraco adulto. EI eyaculado fue obtenido usando el método de la mano enguantada, diluyéndolo inmediatamente en BTS atemperado a $37^{\circ} \mathrm{C}(1: 1$ (vol:vol)). Para el análisis con el "ChemSensor" se tomó una alícuota de $1 \mathrm{~mL}$ de semen sin diluir, que fue congelado a $-80^{\circ} \mathrm{C}$ después de la extracción. Esta congelación se realiza para que los componentes del eyaculado no se vean modificados hasta el momento de su análisis.

Después de su recolección, se evaluaron las características de calidad del eyaculado mediante técnicas habituales de laboratorio. Sólo se seleccionaron para ser congelados aquellos eyaculados que tenían una concentración de $\geq 200 \times 106$ espermatozoides $/ \mathrm{ml}, \geq 85 \%$ de espermatozoides con morfología normal, $\geq 75 \%$ de espermatozoides móviles, $\geq 80 \%$ de espermatozoides viables $\mathrm{y} \leq 1,5 \%$ de espermatozoides con el ADN fragmentado.
Congelación y descongelación de las muestras

Los espermatozoides fueron criopreservados usando el proceso de congelación descrito por Westendorf et al. (1975), y modificado por Thurston et al. (2001) y Carvajal et al. (2004).

Las muestras de semen diluidas fueron enfriadas a $15^{\circ} \mathrm{C}$ durante 2 horas. Posteriormente fueron centrifugadas a $2400 \mathrm{~g}$ durante 3 minutos a 15 ${ }^{\circ} \mathrm{C}$, para la eliminación del diluyente y del plasma seminal. El pellet de espermatozoides obtenido fue de nuevo diluido en el diluyente de congelación FEY (Fructosa-yema de huevo; Thilmant, 1997) hasta una concentración de $1,5 \times 10^{9}$ células $/ \mathrm{mL}$. A continuación se enfriaron las muestras hasta los $5{ }^{\circ} \mathrm{C}$ en un tiempo de 120 minutos en un baño termostático programable, diluyéndolas posteriormente con el diluyente FEY, que esta vez incorporaba glicerol como crioprotector principal, en un porcentaje final del 3\% (pH 6,2, and $1650 \pm 15 \mathrm{mOsm} / \mathrm{kg}$ ), hasta una concentración final de $1 \times 10^{9}$ células $/ \mathrm{mL}$. Las muestras resuspendidas fueron envasadas en pajuelas de $0,5 \mathrm{~mL}$ (Minitüb, Tiefenbach, Alemania), y se congelaron posteriormente en un biocongelador programable usando una curva de congelación adecuada (Carvajal et al., 2004). Las pajuelas se mantuvieron en nitrógeno líquido al menos 2 semanas antes de ser descongeladas. La descongelación de las pajuelas fue realizada en un baño termostático de agua a una temperatura de $37^{\circ} \mathrm{C}$ durante 20 segundos. Así, una vez descongeladas, las muestras fueron diluidas en Beltsville Thawing Solution (BTS; Johnson et al., 1988) (1:1 ( $\mathrm{vol} / \mathrm{vol}), 37^{\circ} \mathrm{C}$ ) e incubadas en el baño termostático a $37{ }^{\circ} \mathrm{C}$ durante 30 minutos, momento en el cual se procedió a el análisis de su calidad. 
Evaluación de la calidad espermática

\section{Medida de la movilidad espermática} post-descongelación

La movilidad espermática fue analizada objetivamente usando un sistema computerizado de análisis del movimiento espermático (Sperm Class Analyzer ${ }^{\circledR}$ Microptic, Barcelona, España), siguiendo el procedimiento descrito por Cremades et al. (2005). Para cada evaluación, tres campos fueron analizados contando un mínimo de 100 espermatozoides por campo. Se determinaron el porcentaje de espermatozoides mótiles totales (\% EMT), mótiles progresivos (\% EMP) y mótiles progresivos rápidos (\% EMPR). Así como los siguientes parámetros de calidad del moviendo: velocidad curvilínea $(\mathrm{VCL}, \mu \mathrm{m} / \mathrm{s})$, velocidad rectilínea (VSL, $\mu \mathrm{m} / \mathrm{s})$, velocidad media (VAP, $\mu \mathrm{m} / \mathrm{s})$, índice de linealidad (LIN, \%), índice de rectitud (STR, \%), índice de oscilación (WOB, \%), amplitud de desplazamiento lateral de la cabeza del espermatozoide $(A L H, \mu m)$ y la media de la frecuencia de batida (BCF, Hz). Sólo el porcentaje de espermatozoides móviles totales (\% EMT) fue considerado para la clasificación por congelabilidad.

\section{Medida de la integridad de la membrana plasmática}

La determinación de la integridad de la membrana espermática fue analizada usando una prueba de doble tinción fluorescente con SYBR-14 y loduro de propidio (IP) (L-7011, Live/Dead Sperm Viability Kit; Molecular Probes Europe, Leiden, Países Bajos), descrito por Garner y Johnson (1995). Las muestras fueron evaluadas mediante un microscopio de fluorescencia (Nikon Eclipse E400, Tokyo, Japón) equipado con un filtro Nikon G-2A (excitación/barrera de 510/590), que permite la excitación simultánea del azul y el verde para el SYBR-14 y el IP respectivamente. Un mínimo de 300 espermatozoides por muestra fueron analizados. Sólo el porcentaje de espermatozoides con la membrana plasmática intacta (\% EMPI) fue considerado en los resultados.

Determinación del grado de fragmentación del ADN espermático

La determinación del grado de fragmentación se realizó, por microscopía de fluorescencia, usando el kit comercial Sperm-Sus-HALOMAX® (ChromaCell, España). Las muestras fueron procesadas de acuerdo a las indicaciones del fabricante. Así brevemente, $25 \mu \mathrm{L}$ de cada una de las muestras descongeladas (diluidas en BTS hasta los $10 \times 10^{6}$ espermatozoides $/ \mathrm{mL}$ ), fueron añadidas al vial comercial de agarosa. Alícuotas de 1,7 mL de las muestras con agarosa se pusieron en el portaobjetos pre-tratados, se cubrieron y se enfriaron a $4^{\circ} \mathrm{C}$ durante 10 minutos. Pasado este tiempo se retiraron los cubres y se sumergieron las muestras en la solución de lisis por 5 minutos. Pasado este tiempo las muestras se lavaron con agua destilada, y se deshidrataron de manera secuencial en baños de etanol (70, 90 y $100 \%)$. Una vez secas las muestras se tiñeron con el fluorocromo adjunto con el kit. Un mínimo de 300 espermatozoides por muestras fueron contados. El porcentaje de espermatozoides fragmentados se calculó en base al tamaño del halo alrededor de la cabeza del espermatozoide.

\section{Análisis con el "ChemSensor"}

Análisis de los compuestos volátiles del semen

Para analizar los compuestos volátiles del semen, se utilizó una alícuota de $1 \mathrm{~mL}$ de semen completo, que se homogenizó y se colocó en el vial de análisis. Los análisis se realizaron en un "ChemSensor" (cromatógrafo de gasesmasas CG 6890, Agilent Technologies, Palo Alto, CA, EE. UU., acoplado al espacio de cabeza dinámico). Las muestras fueron inyectadas automáticamente y retenidas en la trampa 
del espacio de cabeza dinámico, utilizando tres ciclos de purga/trampa (TurboMatrix HS40 con Air Monitoring Trap, PerkinElmer, Waltham, MA, EE.UU.). A continuación, las muestras fueron desorbidas térmicamente $\left(280^{\circ} \mathrm{C}\right)$ de la trampa y fueron inyectadas $\left(200^{\circ} \mathrm{C}\right)$ en el equipo a través de una columna capilar (HP-5MS: $30 \mathrm{~m} \times$ 0,32 mm i.d., 0,25 $\mu \mathrm{m}$ film de espesor). El espectrómetro de masas (MS 5973MSD) constaba de un detector de cuadrupolo operando en modo de impacto electrónico, con una energía de ionización de $70 \mathrm{eV}$, y línea de transferencia de $110^{\circ} \mathrm{C}$. El escaneo de masas se realizó en el rango de fragmentos iónicos m/z = 50-200 a.m.u./q.

Los diferentes volátiles obtenidos fueron plasmados en un cromatograma, e identificados usando la librería Wiley del propio equipo, considerando que el compuesto identificado era aquél que poseía el mayor porcentaje de probabilidad, descartándose los que tuvieran una probabilidad inferior al $50 \%$.

\section{Análisis quimiométrico}

Una vez analizados los compuestos volátiles del semen se utilizaron los resultados de las diferentes fracciones másicas para ser analizadas con el sistema quimiométrico. El sistema de tratamiento de datos fue el integrado en el programa informático. Los análisis multivariantes se realizaron sobre los datos normalizados, utilizando el software quimiométrico ( $\mathrm{Pi}$ rouette 3.11, Infometrix Inc). La clasificación predictiva de las muestras, de acuerdo con la congelabilidad de las muestras (2 grupos, buenos y malos congeladores), se realizó mediante el método de reconocimiento de patrones (Soft Independent Modelling Class Analogy, SIMCA). En este método cada clase o grupo (en este caso grupos de congelabilidad) se modela usando un análisis de com- ponentes principales de forma independiente, de tal manera que cada grupo tiene un modelo específico, que lo describe el número óptimo de componentes principales (en este caso estos componentes serían las fracciones másicas). De esta manera se puede construir un espacio para cada grupo, cuyo volumen marca el límite entre las muestras pertenecientes a cada uno los grupos de estudio y las muestras que no pertenecen a ninguno, para un nivel de confianza de $95 \%$.

Los resultados obtenidos con este modelo se expresan de dos maneras. La primera mediante la clasificación predicha o esperada de las muestras, o lo que es lo mismo el modelo nos indica el número de muestras que ha podido clasificar dentro de cada grupo o en ninguno de ellos, describiéndonos el número de muestras bien clasificadas o el número de falsos positivos (animales que congelan mal, clasificados como buenos congeladores) y el número de falsos negativos (animales buenos congeladores clasificados como malos congeladores). El segundo por las distancias ortogonales de las muestras del conjunto respecto a las de las dos clases o grupos de congelabilidad definidos por el modelo. De forma visual estas distancias de separación entre los grupos se representan mediante un diagrama de Coomans (Coomans et al., 1984) (Figura 1), donde observamos la buena o mala clasificación de las muestras observando su posición respecto a los grupos, siendo el eje $X$ para los malos congeladores, y el eje Y para los buenos congeladores. Así cuanto más próximos al cuadrante superior izquierda (o dentro de este) estén los malos congeladores, y más próximos al cuadrante inferior derecho (o dentro de este) estén los buenos congeladores, mayor serán las distancias ortogonales entre los grupos y por tanto la validez de nuestro modelo. 


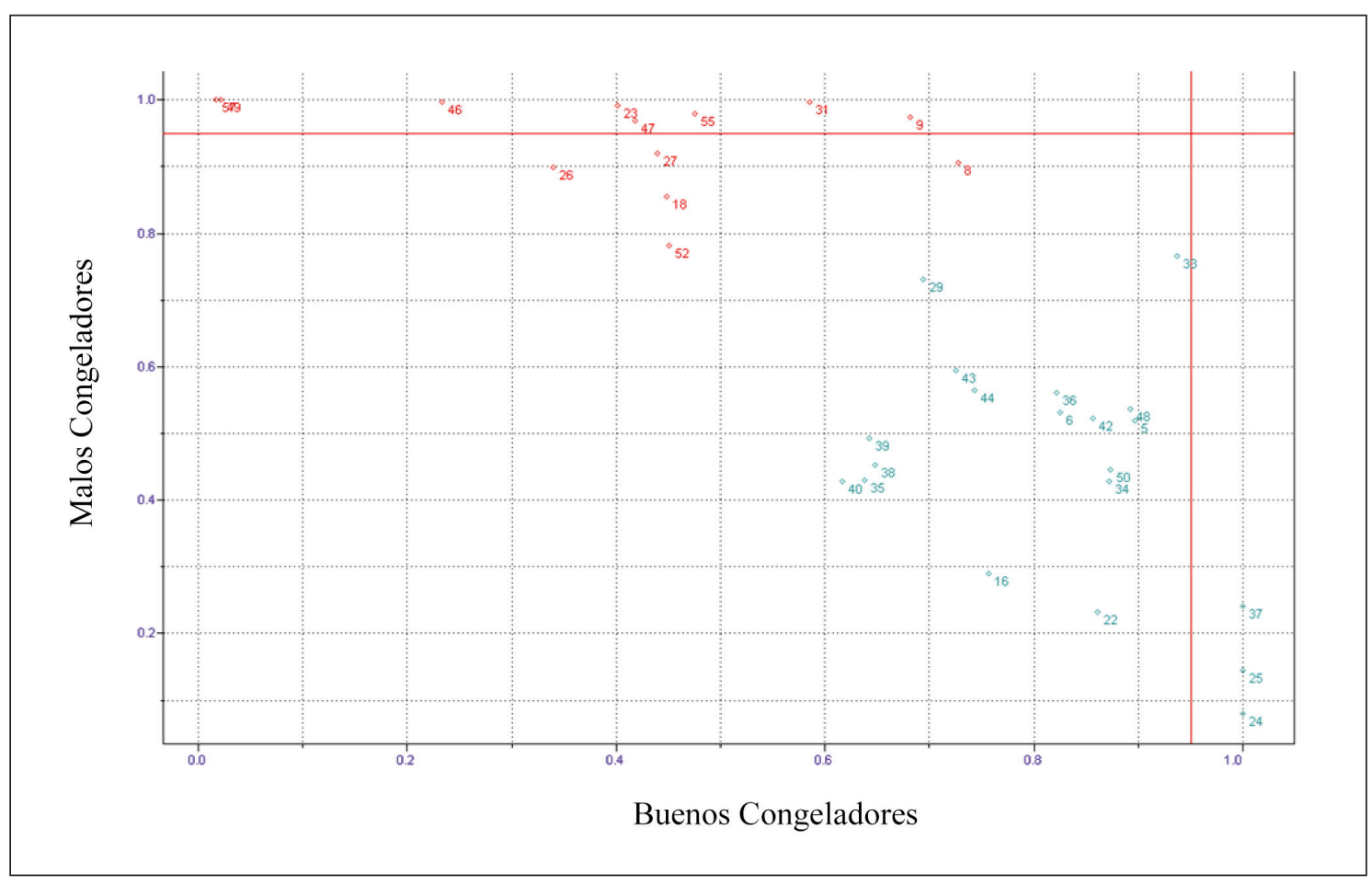

Figura 1. Diagrama de Coomans. Representación visual de las distancias de separación entre los grupos de congelabilidad (buenos (azul) y malos (rojo) congeladores).

Las coordenadas de la gráfica son los valores predictivos de los grupos de congelabilidad (buenos y malos congeladores) en función del modelo de componentes principales de cada uno de los grupos (X para los buenos congeladores; e Y para los malos congeladores).

Las líneas de color rojo muestran 4 cuadrantes, y son la representación de las distancias críticas que se corresponden con el intervalo de confianza del 95\%

Figure 1. Coomans diagram, visual representation of the separation distances between freezability groups (good (blue) and bad (red) freezers).

Análisis de la sensibilidad y especificidad del modelo

El análisis de la sensibilidad y especificidad, es usado para determinar la validez de un modelo frente a la presencia de falsos positivos y falsos negativos en los modelos de diagnostico de enfermedades. Para nuestro caso consideraremos que ser buen congelador es positivo y ser mal congelador es negativo. Así la sensibilidad nos indicará la capacidad de nuestro modelo para determinar que un ve- rraco es un buen congelador cuando realmente lo es, y la especificidad nos indica la capacidad de nuestro modelo para determinar que un macho congelará mal cuando realmente es un mal congelador. Así una alta sensibilidad nos indicará que nuestro modelo es capaz de determinar correctamente los buenos congeladores, y una alta especificidad nos indicará la capacidad de nuestro modelo para determinar correctamente si un verraco es mal congelador. 
Análisis estadístico

Para la clasificación de los diferentes eyaculados en función de su congelabilidad, se realizó un análisis de clasificación por clusters o grupos con el programa estadístico Statgraphics (Versión 15.2.12., StatPoint Technologies, Inc. Warrenton, Virginia, EE. UU.), usando como criterio de clasificación la media del grupo, donde la distancia entre dos clústers fue la distancia media de las observaciones de un clúster a la del otro. Y la distancia métrica entre clústers usada fue la euclidiana al cuadrado, que es la suma de los cuadrados de las diferencias de los valores de las observaciones en los grupos. Como variables de discriminación se usaron tan solo el número de espermatozoides vivos totales y el porcentaje de espermatozoides móviles totales a los 30 minutos post-descongelación, debido a que la inclusión de más parámetros afectaba al sistema estadístico de clasificación, diminuyendo notablemente el R-cuadrado de nuestro modelo, y por tanto la validez del mismo. Además de forma general ya esta establecido por otros autores que el uso de estas dos variables es suficiente para una adecuada clasificación de los eyaculados para su congelabilidad (Roca et al., 2006; Barranco et al., 2013). Las diferencias entre las medias de los grupos fueron contrastadas con el paquete estadístico SAS (Versión 9.0, SAS Institute Inc., Cary, NC, EE. UU.) mediante un análisis GLM, para determinar si dichas diferencias entre grupos eran significativas $(P<0,05)$. Los resultados se expresan como medias con su respectivo Error Estándar de la Media (EEM). Fueron consideradas las diferencias significativas a $\mathrm{P}<0,05$.

La clasificación realizada con el sensor químico se hizo mediante el programa informático Pirouette (Versión 3.10., Infometrics, INC, EE. UU.) usando el sistema de análisis quimiométrico SIMCA (Soft Independent Modelling Class Analogy), como se ha descrito anterior- mente. Y el análisis de la sensibilidad y especificidad se realizo mediante el programa Excel (Microsoft® Office Excel 2003).

\section{Resultados}

La clasificación de los diferentes eyaculados en función de su congelabilidad, según el \% EMPI y el \% EMT se muestra en la Tabla 1. En estos resultados se puede observar que 20 de los 33 eyaculados son considerados como pertenecientes a machos buenos congeladores, y 13 de los 33 a machos malos congeladores. Una vez fueron clasificadas las muestras de acuerdo a su congelabilidad, se relacionaron con los resultados obtenidos de análisis de los compuestos volátiles.

Resultados del análisis de los volátiles del semen

No se encontraron diferencias significativas en el porcentaje de los diferentes volátiles detectados e identificados entre los grupos de congelación $(P>0,05)$ (Tabla 2). El compuesto volátil mayoritario fue el hexano, con un porcentaje de área respecto del total de áreas del $93 \%$. Compuestos como octametilciclotetrasiloxano, decametil ciclopentasiloxano, dodecametil ciclohexasiloxano y tetradecametil ciclopentasiloxano se identificaron como residuos de la propia columna cromatográfica con un porcentaje medio de $2-3 \%$. El resto de componentes estuvieron presentes en una proporción menor de $0,5 \%$.

Resultados del análisis quimiométrico

En la Tabla 3 se puede observar como en función de la clasificación por congelabilidad, el modelo nos da una clasificación predicha o esperada de las diferentes muestras analizadas. Así puede observarse que los animales 
Tabla 1. Valores medios de la calidad espermática pre-congelación y post-descongelación de los distintos grupos de congelabilidad

Table 1. Mean values of pre-freeze and post-thaw sperm quality of the different freezability groups

\begin{tabular}{llll}
\hline Variables & Buenos congeladores & Malos congeladores & EEM \\
\hline \multicolumn{4}{c}{ Pre-Congelación } \\
$\operatorname{EMPI}^{2}(\%)$ & 80,9 & 84,2 & 2,75 \\
$\operatorname{EAF}^{3}(\%)$ & 0,82 & 0,99 & 1,2 \\
$\operatorname{EMT}^{4}(\%)$ & 78,75 & 81 & 2,4 \\
\hline & & Post-Descongelación & \\
$\operatorname{EMPI}^{2}(\%)$ & $57,8^{\text {a }}$ & $42,1^{\mathrm{b}}$ & 1,32 \\
$\operatorname{EAF}^{3}(\%)$ & 1,1 & 1,3 & 0,95 \\
$\operatorname{EMT}^{4}(\%)$ & $50,3^{\mathrm{a}}$ & $28,5^{\mathrm{b}}$ & 1,42 \\
$\operatorname{EMP}^{5}(\%)$ & $41,3^{\mathrm{a}}$ & $24,8^{\mathrm{b}}$ & 1,4 \\
$\operatorname{EMPR}^{6}(\%)$ & $39,6^{\mathrm{a}}$ & $22,8^{\mathrm{b}}$ & 1,5 \\
$\operatorname{VCL}^{7}(\mu \mathrm{m} / \mathrm{s})$ & 106,9 & 97,6 & 3,4 \\
$\operatorname{VSL}^{8}(\mu \mathrm{m} / \mathrm{s})$ & 75,4 & 72,7 & 3,06 \\
$\operatorname{VAP}^{9}(\mu \mathrm{m} / \mathrm{s})$ & 93,3 & 85,2 & 3 \\
$\operatorname{LIN}^{10}(\%)$ & 70,7 & 74,4 & 2,02 \\
$\operatorname{STR}^{11}(\%)$ & 80,9 & 85,1 & 1,93 \\
$\operatorname{WOB}^{12}(\%)$ & 87,2 & 87,4 & 1,09 \\
$\operatorname{ALH}^{13}(\mu \mathrm{m})$ & 2,91 & 2,59 & 0,12 \\
$\operatorname{BCF}^{14}(\mathrm{~Hz})$ & 7,68 & 7,87 & 0,15 \\
$\mathrm{~N}^{15}$ & 20 & 13 & \\
\hline
\end{tabular}

${ }^{1}$ Error estándar de la media; ${ }^{2}$ porcentaje de espermatozoides con la membrana plasmática intacta; ${ }^{3}$ porcentaje de espermatozoides con el ADN fragmentado; ${ }^{4}$ porcentaje de espermatozoides móviles totales; ${ }^{5}$ porcentaje de espermatozoides mótiles progresivos; ${ }^{6}$ porcentaje de espermatozoides mótiles progresivos rápidos; ${ }^{7}$ velocidad curvilínea, ${ }^{8}$ velocidad rectilínea, ${ }^{9}$ velocidad media; ${ }^{10}$ índice de linealidad; 11 índice de rectitud; ${ }^{12}$ índice de oscilación; ${ }^{13}$ amplitud de desplazamiento lateral de la cabeza del espermatozoide; ${ }^{14}$ media de la frecuencia de batida; ${ }^{15}$ Número de machos incluidos en cada grupo.

Letras diferentes en la misma variable indican diferencias significativas $(P<0,05)$. 
Tabla 2. Porcentaje del área respecto del total de áreas de los distintos compuestos volátiles identificados en la muestras de semen

Table 2. Percentage of area with respect to total area from the individual volatile compounds identified in the semen samples

\begin{tabular}{lccc}
\hline Compuesto Volátil & $\begin{array}{c}\text { Buenos congeladores } \\
(\mathrm{n}=20)\end{array}$ & $\begin{array}{c}\text { Malos congeladores } \\
(\mathrm{n}=13)\end{array}$ & EEM $^{1}$ \\
\hline Hexano & 92,45 & 92,56 & 0,81 \\
Hexanol & 0,2 & 0,09 & 0,071 \\
Benzaldehído & 0,43 & 0,43 & 0,003 \\
Octametil Ciclotetrasiloxano & 1,55 & 1,53 & 0,109 \\
Nonanal & 0,98 & 1,18 & 0,36 \\
Decametil Ciclopentasiloxano & 1,3 & 1,27 & 0,103 \\
Dodecametil Ciclohexasiloxano & 1,64 & 1,57 & 0,135 \\
Trimetoxi-silano de Propilo & 0,22 & 0,18 & 0,035 \\
Tetradecametil Ciclopentasiloxano & 1,01 & 1 & 0,082 \\
\hline
\end{tabular}

${ }^{1}$ Error estándar de la media.

Tabla 3. Clasificación predicha o esperada de las diferentes muestras analizadas en función de su congelabilidad y distancia ortogonal entre los dos grupos de congelabilidad Table 3. Predicted or expected classification from the various samples analyzed according to their freezability and orthogonal distance between the two groups of freezability

\begin{tabular}{lcccc}
\hline & $\begin{array}{c}\text { Malos } \\
\text { predichos }\end{array}$ & $\begin{array}{c}\text { Buenos } \\
\text { predichos }\end{array}$ & $\begin{array}{c}\text { No } \\
\text { clasificados }\end{array}$ & $\begin{array}{c}\text { Distancia entre los } \\
\text { grupos de congelabilidad }\end{array}$ \\
\hline Malos congeladores & 12 & 1 & 0 & 2,62 \\
Buenos congeladores & 0 & 20 & 0 & \\
\hline
\end{tabular}

clasificados como buenos congeladores son discriminados de los malos congeladores. En cambio, en el grupo de los malos congeladores aparece un animal clasificado como bueno, creando así un falso positivo. No se observaron falsos negativos, ni hubo muestras que no pudieran ser clasificadas.

La distancia ortogonal de las muestras del conjunto respecto a la de los dos grupos de con- gelabilidad definidos por el modelo fue de 2,62 (Tabla 3). Al observar dichas distancias, puede determinarse que éstas son relativamente bajas, ya que para que se consideren diferentes significativamente, la distancia debería ser de al menos 3. Pero a pesar de ello, el modelo muestra un óptimo poder clasificatorio, como puede observarse en el diagrama de Coomans (Figura 1), donde se representan 
cada una de las muestras analizadas en función de si son malos congeladores (eje $X$ ) o buenos congeladores (eje Y), observándose los malos congeladores de color rojo cerca del cuadrante superior izquierdo, separados de los buenos congeladores de color azul, cerca del cuadrante inferior derecho.

Resultados del análisis de sensibilidad y especificidad

Los resultados predichos o estimados de nuestro modelo determinaron que hubo un falso positivo, es decir, un macho mal congelador que se clasificó como buen congelador. Para determinar, la validez del modelo se calculó la sensibilidad y especificidad del mismo. Así la sensibilidad es la división entre el número de animales buenos congeladores reales entre la resta del número de animales buenos congeladores reales más los falsos negativo. Por tanto el valor de la sensibilidad fue de 1 al no existir falsos negativos. Y la especificidad es el número de malos congeladores reales, divididos entre el número de malos congeladores reales más los falsos positivos (hubo uno), siendo el valor de 0,93. Así estos resultados muestran que nuestro modelo tiene una elevada sensibilidad y una alta especificidad lo cual nos constata la robustez de nuestro modelo en la predicción de la congelabilidad.

\section{Discusión}

Muchos investigadores han centrado sus estudios en determinar las diferencias entre un eyaculado de un macho buen congelador, y un eyaculado de un macho mal congelador (Thurston et al., 2002; Maldjian et al., 2005; Harshan et al., 2006; Esteso et al., 2006; Hernández et al., 2006; Casas et al., 2010a). Pero pocos han buscado un método que permita una discriminación precoz de los eyaculados en función de su futura congelabilidad (Petrunkina et al., 2004; Gil et al., 2005; Casas et al., 2009 y 2010b). La posibilidad de obtener un modelo capaz de determinar la congelabilidad de un eyaculado sin tener que congelarlo, descongelarlo y valorarlo, sería un gran avance para la utilización del semen congelado en la inseminación artificial porcina, ya que permitiría determinar qué animales son los más adecuados para congelar sus espermatozoides y garantizar una mayor calidad a la descongelación, pudiendo así rentabilizar los machos de alto interés genético y crear óptimos bancos de espermatozoides congelados.

El estudio de los componentes volátiles usando una "pseudo-nariz electrónica" o "ChemSensor" es una técnica habitual en la investigación de la calidad de los alimentos y la clasificación de muestras (Olsson et al., 2000; Radovic et al., 2001; Ansorena et al., 2001; Landaud et al., 2008; Rodríguez-Bencomo et al., 2009). El ChemSensor permite determinar qué compuestos aromáticos tiene una muestra biológica (Radovic et al., 2001; Ansorena et al., 2001; Landaud et al., 2008) y, usando esos compuestos, clasificar diferentes tipos de muestras independientemente de su origen en aspectos muy diferentes, como puede ser el estado de curación de ciertos alimentos (Ruiz et al., 1999; Martín et al., 2009), la calidad organoléptica (Rodríguez-Bencomo et al., 2009) o discriminar muestras contaminadas de otras que no lo están (Olsson et al., 2000), constituyendo el uso de esta herramienta una técnica de discriminación y clasificación muy eficaz. Así la clara capacidad de discriminación que poseen equipos como el "ChemSensor" usando como matriz muestras biológicas tan diferentes, nos hizo pensar que el estudio de los componentes volátiles del semen podría servir como un método para determinar de una forma rápida y precisa, si una muestra de semen sería apta o no para su criopreservación, tratando de correlacionar la proporción de alguno de estos volátiles con la congelabilidad del macho. 
Los resultados obtenidos muestran que los componentes volátiles detectados en semen de porcino son muy escasos, en comparación con los obtenidos en análisis de otras muestras biológicas (Ansorena et al., 2001; Radovic et al., 2001; Martín et al., 2009; RodríguezBencomo et al., 2009), determinándose que los componentes volátiles detectados no aportan la información necesaria para poder usarlos como una herramienta de clasificación en sí misma de la futura congelabilidad de los eyaculados. Sin embargo el estudio de la clasificación de los eyaculados por su congelabilidad no termina con los resultados de los cromatogramas obtenidos de las muestras, sino que el "ChemSensor" utiliza el espectro total de masas obtenido en estos análisis, representando cada fracción másica resultante de la separación por cromatografía. Así cada fracción másica puede ser considerada como un potencial descriptor químico de una muestra y el conjunto de fracciones másicas es sujeto a un análisis multivariante (SIMCA) para, de esta manera, obtener información cuantitativa o cualitativa que pueda predecir la calidad de una muestra (Laguerre et al., 2007). En este estudio se utilizó el espectro total de fracciones másicas obtenidas de cada perfil de compuestos volátiles de las muestras de semen analizadas, clasificándolas previamente por grupos de congelabilidad, para tratar de realizar un análisis discriminatorio de cada una de las muestras usando el método SIMCA. Los resultados obtenidos permitieron el uso de hasta 500 fracciones másicas como variables de discriminación de las muestras, entre los diferentes grupos (buenos y malos congeladores).

El modelo que se obtiene con este estudio nos muestra que es capaz de discriminar los eyaculados de los buenos de los malos congeladores, determinándose que los animales clasificados como buenos congeladores nunca se clasifican como malos, es decir que no existen falsos positivos. Teniendo en cuenta los resultados observados de sensibilidad y especificidad, al ser estos valores muy elevados (la sensibilidad del modelo es de 1, y la especificidad de 0,93 ), este modelo nos indica que la probabilidad de que haya falsos positivos es muy baja y menor aun de que existan falsos negativos.

Al observar los resultados de las distancias ortogonales entre grupos los congelabilidad, si nos fijamos en el diagrama de Coomans (Figura 1) el modelo muestra bien separados los buenos de los malos congeladores, pero no con una discriminación perfecta, que sería cuando los malos congeladores estuvieran todos enmarcados en el cuadrante superior izquierdo, y los buenos congeladores en el cuadrante inferior derecho. Para que las distancias entre grupos sean significativas su valor debe ser mayor de 3 (Busto et al., 2002), pero nuestro estudio marca dichas distancias en un valor de 2,62. Este valor podría ser insuficiente para algunos autores, pero autores como Laguerre et al. (2007) determinan que distancias de separación entre grupos por encima de 2 se pueden considerar significativas si el número de muestras del que se parte es bajo. Por tanto, en nuestro caso, se puede considerar que la distancia inter-clase obtenida con este modelo sería significativa, ya que se trata de un estudio preliminar, donde se han utilizado 33 muestras de semen de cerdo Ibérico, siendo este valor bajo para poder crear un modelo matemático estable, pero suficientes para un estudio preliminar que buscaba determinar si este tipo de análisis servirían para poder discriminar muestras de semen por su grado de congelabilidad.

La capacidad de discriminación que ha mostrado este modelo usando semen completo nos indica la existencia de diferencias en la composición del semen de buenos y malos congeladores. Pero se desconoce cuales son dichas diferencias. El "ChemSensor" aporta información de discriminación con el uso de múltiples variables, las cuales en este caso son 
fracciones másicas obtenidas del análisis de los compuestos volátiles del semen. Sin embargo es difícil la identificación individual de los componentes químicos responsables de cualquiera de las diferencias observadas (Berlioz et al., 2006), y por tanto determinar a qué compuestos o componentes corresponden estas fracciones es una labor complicada, más todavía a falta de algún trabajo referencial que analice la composición de fracciones másicas del semen.

Las diferencias entre los eyaculados de machos buenos y malos congeladores en lo que se refiere a su composición han sido estudiadas por múltiples autores. Algunos autores atribuyen que la mayor o menor resistencia a la congelabilidad viene determinada por la composición lipídica de la membrana del espermatozoide. Así la presencia de ácidos grasos poliinsaturados confiere una gran fluidez a la membrana la cual podría dar una mejor resistencia al daño surgido por la formación de cristales de hielo durante la congelación (Maldjian et al., 2005; Waterhouse et al., 2006). O variaciones en la proporción de algunos ácidos grasos también se relacionan con la criotolerancia en el semen (Miller et al., 2005). Mientras que otros autores muestran resultados de que es la composición proteica de la membrana la responsable de esta congelabilidad (Casas et al., 2009 y 2010b).

Otro grupo de autores sin embargo, afirman que la mayor o menor resistencia a la congelación viene determinada por la composición del plasma seminal, ya sea por la presencia de lípidos en el plasma seminal en forma de gránulos y vesículas membranosas (Piehl et al., 2006) denominadas como prostasomas, que contienen gran cantidad de colesterol y esfingomielina, y que podrían estar envueltas en la estabilización de la membrana espermática y por tanto jugar un papel importante en la respuesta a la crioconser- vación espermática (He et al., 2001). O debido a la composición en proteínas del mismo (Hernández et al., 2007; Singh et al., 2014).

En cualquier caso parece no existir un único factor que determine la congelabilidad de una muestra (Hernández et al., 2007), sino que puede ser un conjunto de factores los que determinen la futura resistencia de un eyaculado a su congelación; factores entre los que se podría incluir tanto la composición del plasma seminal como la composición de la membrana del espermatozoide. Así nuestro modelo predictivo, aunque preliminar, se muestra como un modelo eficaz, ya que incluye tanto los espermatozoides como el plasma seminal, y por tanto engloba los factores que se han determinado como posibles responsables de la criotolerancia de semen. Cuales de estos factores son los que engloban el modelo es muy difícil de discernir, y menos sin otros trabajos que estudien aspectos similares. Por tanto se debería determinar con más exactitud los componentes volátiles del semen y de donde proceden, ya que muchos de estos compuestos son producidos a partir ácidos grasos (Frankel, 1972; Forss, 1972), o pueden estar asociados a proteínas (Pérez, 2006), y por tanto se podrán llegar a relacionar con los resultados de congelabilidad encontrados por otros autores.

Por todo esto se puede concluir que el "ChemSensor" se muestra como una posible herramienta adecuada para la discriminación precoz entre buenos y malos congeladores. El uso de este equipo para este tipo de muestras biológicas es del todo novedoso, y no ha sido descrito nunca en el semen de porcino, pero se trata de un trabajo preliminar y sería necesario aumentar el número de muestras analizadas para poder obtener un modelo más eficaz, con mayores distancias de discriminación y sin falsos positivos. 


\section{Agradecimientos}

Queremos dedicar este trabajo al Dr. Atanasio Carrasco Manzano que dedicó gran parte de su vida profesional a la mejora de las técnicas discriminantes mediante el "ChemSensor" en diferentes matrices. Desgraciadamente no ha podido ver finalizada esta publicación, falleciendo un mes antes de haberla concluido. Sirvan estas líneas de recuerdo y homenaje a una extraordinaria persona y ejemplo para la profesión investigadora.

\section{Bibliografía}

Ansorena D, Gimeno O, Astiasarán I, Bello J (2001). Analysis of volatile compounds by GC-MS of a dry fermented sausage: chorizo de Pamplona. Food Research Internacional 34: 67-75.

Armanino C, Casolino MC, Casale M, Forina M (2008). Modelling aroma of three Italian red wines by headspace-mass spectrometry and potencial functions. Analytica Chimica Acta 614: 134-142.

Barranco I, Ortega MD, Martínez-Alborcia M, Vázquez JM, Martínez E, Roca J. (2013) Season of ejaculate collection influences the freezability of boar spermatozoa. Cryobiology 67: 299-304.

Berlioz B, Cordella C, Cavalli JF, Lizzani-Cuvelier L, Loiseau AM, Fernández X (2006). Comparison of the Amounts of Volatile Compounds in French Protected Designation of Origin Virgin Olive Oils. Journal of the Agricultural Food Chemistry 54: 10092-10101.

Busto O, Martí MP, Guasch J (2002). La nariz electrónica en el control de calidad de vinos. Nuevas aplicaciones. Tecnología del vino: Tratamientos y equipos para viticultura y enología 5: 31-35.

Carrasco JA, Duque JP (2013). Classification of Iberian pigs according to intensive feeding by ChemSensor. Aceites y Grasas 64: 166-172.

Carvajal G, Cuello C, Ruiz M, Vázquez JM, Martínez EA, Roca J (2004). Effects of centrifugation before freezing on boar sperm cryosurvival. Journal of Andrology 25: 389-396.
Casas I, Sancho S, Briz M, Pinart E, Bussalleu E, Yeste M, Bonet S (2009). Freezability prediction of boar ejaculates assessed by functional sperm parameters and sperm proteins. Theriogenology 72: 930-948.

Casas I, Sancho S, Briz M, Pinart E, Bussalleu E, Yeste M, Bonet S (2010a). Fertility after postcervical artificial insemination with cryopreserved sperm from boar ejaculates of good and poor freezability. Animal Reproduction Science 118: 69-76.

Casas I, Sancho S, Ballester J, Briz M, Pinart E, Bussalleu $E$, Yeste M, Fàbrega $A$, Rodríguez-Gil JE, Bonet S (2010b). The HSP90AA1 sperm content and the prediction of the boar ejaculate freezability. Theriogenology 74: 940-950.

Cremades T, Roca J, Rodríguez-Martínez H, Abaigar T, Vázquez JM, Martínez EA (2005). Kinematic changes during the cryopreservation of boar spermatozoa. Journal of Andrology 26: 610-618.

Cynkar W, Dambergs RG, Smith P, Cozzolino D (2010). Classification of Tempranillo wines according to geographic origin: Combination of mass spectrometry based electronic nose and chemometrics. Analytica Chimica Acta 660: 227-231.

Coomans D, Broeckaert I, Derde MP, Tassin A, Massart DL, Wold S (1984). Use of a microcomputer for the definition of multivariate confidence regions in medical diasnosis based on clinical laboratory profiles. Computers and Biomedical Research 17: 1-14.

Esteso MC, Soler AJ, Fernández-Santos MR, Quintero-Moreno AA, Garde JJ (2006). Functional significance of the sperm head morphometric size and shape for determining freezability in iberian red deer (Cervus elaphus hispanicus) epididymal sperm samples. Journal of Andrology 27: 662-670.

Forss DA (1972). Odor and flavor compounds from lipids. Progress in the Chemistry o Fats and Other Lipids 13: 181-258.

Frankel EN (1972). Review. Recent advances in lipid oxidation. Journal of the Science of Food and Agriculture 54: 495-511. 
Garner DL, Johnson LA (1995). Viability Assessment of Mammalian Sperm Using SYBR-14 and Propidium lodide. Biology of Reproduction 53: 276-284.

Gil MA, Roca J, Cremades T, Hernández M, Vázquez JM, Rodríguez-Martínez H, Martínez EA (2005). Does multivariate analysis of post-thaw sperm characteristics accurately estimate in vitro fertility of boar individual ejaculates? Theriogenology 64: 305-316.

Harshan HM, Singh LP, Arangasamy A, Ansari MR, Kumar S. (2006). Effect of buffalo seminal plasma heparin binding protein (HBP) on freezability and in vitro fertility of buffalo cauda spermatozoa. Animal Reproduction Science 93: 124-133.

He L, Bailey JL, Buhr MM (2001). Incorporating lipids into boar sperm decreases chilling sensitivity but not capacitation potencial. Biology of Reproduction 64: 69-79.

Hernández M, Roca J, Ballester J, Vázquez JM, Martínez EA, Johannisson A, Saravia F, Rodríguez-Martínez H (2006). Differences in SCSA outcome among boars with different sperm freezability. International Journal of Andrology 29: 583-591.

Hernández M, Roca J, Calvete JJ, Sanz L, MuiñoBlanco T, Cebrián-Pérez JA, Vázquez JM, Martínez EA (2007). Cryosurvival and in vitro fertilizing capacity postthaw is improved when boar spermatozoa are frozen in the presence of seminal plasma from good freezer boars. Journal of Andrology 28: 689-697.

Holt WV, Medrano A, Thurston LM, Watson PF (2005). The significance of cooling rates and animal variability for boar sperm cryopreservation: insights from the cryomicroscope. Theriogenology 63: 370-382.

Johnson LA, Aalbers JG, Grooten HIG (1988). Artificial insemination of swine: fecundity of boar stored in beltsville ts (bts), modified modena ( $\mathrm{mm}$ ) or $\mathrm{mr}-\mathrm{a}$ and inseminated on one, three and four days after collection. Zuchthygiene 23: 49-55.

Laguerre M, Mestres C, Davrieux F, Ringuet J, Boulanger R (2007). Rapid discrimination of scented rice by solid-phase mMicroextraction, mass spectrometry, and mMultivariate analysis used as a mass sensor. Journal of Agricultural and Food Chemistry 55: 1077-1083.

Landaud S, Helinck S, Bonnarme P (2008). Formation of volatile sulfur compounds and metabolism of methionine and other sulfur compounds in fermented food. Applied Microbiology and Biotechnology 77: 1191-1205.

Maldjian A, Pizzi F, Gliozzi T, Cerolini S, Penny P, Noble R (2005). Changes in sperm quality and lipid composition during cryopreservation of boar semen. Theriogenology 63: 411-421.

Martín C, Lizaso J, Mallo JJ, Carrasco JA, Gimenez L, Gómez E, Rodríguez A, de Mercado E, Sanz E (2009). Diferenciación en la calidad del cerdo Ibérico de cebo. Tierras de Castilla y León 154: 94-98.

Medrano A, Watson PF, Holt WV (2002). Importance of cooling rate and animal variability for boar sperm cryopreservation: insights from the cryomicroscope. Reproduction 123: 315-322.

Miller RRJr, Cornett CL, Waterhouse KE, Farstad W (2005). Comparative aspects of sperm membrane fatty acid composition in silver (Vulpes vulpes) and blue (Alopex lagopus) foxes, and their relationship to cell cryopreservation. Cryobiology 51: 66-75.

Olsson J, Böerjesson T, Lundstedt T, Schnürer J (2000). Volatiles for mycological quality grading of barley grains: determinations using gas chromatography-mass spectrometry and electronic nose. International Journal of Food Microbiology 59: 167-178.

Pérez M (2006). Interacción de los compuestos aromáticos del jamón con la matríz proteica. Tesis Doctoral. Universidad Politécnica de Valencia.

Peris M, Escuder-Gilabert L (2009). A 21st century technique for food control: Electronic noses. Analytica Chimica Acta 638: 1-15.

Petrunkina AM, Gröpper B, Günzel-Apel AR, Töpfer-Petersen $E$ (2004). Functional significance of the cell volume for detecting sperm membrane changes and predicting freezability in dog semen. Reproduction 128: 829-842. 
Piehl L, Cisale H, Torres N, Capani F, Sterin-Speziale N, Hager A (2006). Biochemical characterization and membrane fluidity of membranous vesicles isolated from boar seminal plasma. Animal Reproduction Science 92: 401-410.

Radovic BS, Careri M, Mangia A, Musci M, Gerboles M, Anklam E (2001). Contribution of dynamic headspace GC-MS analysis of aroma compounds to authenticity testing of honey. Food Chemistry 72: 511-520.

Roca J, Hernández M, Carvajal G, Vazquez JM, Martinez EA (2006). Factors influencing boar sperm cryosurvival. Journal of Animal Science 84: 2692-2699.

Rodríguez-Bencomo JJ, Ortega-Heras M, PérezMargariño S, Gonzáñez-Huerta C (2009). Volatile compounds of red wines macerated with Spanish, American, and French oak chips. Journal of Agricultural and Food Chemistry 57: 6383-6391.

Ruiz J, Ventanas J, Cava R, Andrés A, García C (1999). Volatile compounds of dry-cured Iberian ham as affected by the length of the curing process. Meat Science 52: 19-27.

Singh M, Ghosh SK, Prasad JK, Kumar A, Tripathi RP, Bhure SK, Srivastava N (2014). Seminal PDC109 protein vis-à-vis cholesterol content and freezability of buffalo spermatozoa. Animal Reproduction Science 144: 22-29.

Thilmant P (1997). Congélation du sperme de verrant en paillette de $0,5 \mathrm{~mL}$. Résultats sur le terrain. Annales de Médecine Vétérinaire 141: 457-462.

Thurston LM, Watson PF, Holt WV (1999). Sources of variation in boar spermatozoa fertility following cryopreservation. Journal of Reproduction and Fertility 117: 271-280.
Thurston LM, Watson PF, Mileham AJ, Holt WV (2001). Morphologically distinct sperm subpopulations defined by Fourier shape descriptors in fresh ejaculate correlate with variation in boar semen quality following cryopreservation. Journal of Andrology 22: 382-394.

Thurston LM, Siggins K, Mileham AJ, Watson PF, Holt WV (2002). Identification of amplified restriction fragment length polymorphism markers linked to genes controlling boar sperm viability following cryopreservation. Biology of Reproduction 66: 545-554.

Vera L, Mestres M, Boqué R, Busto O, Guasch J (2010). Use of synthetic wine for models transfer in wine analysis by HS-MS e-nose. Sensors and Actuators, B: Chemical 143: 689-695.

Waterhouse KE, Hofmo PO, Tverdal A, Miller RRJr. (2006). Within and between breed differences in freezing tolerance and plasma membrane fatty acid composition of boar sperm. Reproduction 131: 887-894

Watson PF (1995). Recent developments and concepts in the cryopreservation of spermatozoa and the assessment of their post-thawing function. Reproduction Fertility and Development 7: 871-891.

Vishwanath R (2003). Artificial insemination: the state of the art. Theriogenology 59: 571-584.

Westendorf P, Richter L, Treu H (1975). Zur Tiefgefrierung von Ebersperma. Labor- und Besamungsergebnisse mit dem Hülsenberger PaillettenVerfahren. Deutsche Tierarztliche Wochenschrift 82: 261-267.

(Aceptado para publicación el 10 de julio de 2014) 